\title{
Predicting Temporal Intention in Resource Sharing
}

\author{
Hany M. SalahEldeen \\ Old Dominion University \\ Norfolk, VA, USA \\ hany@cs.odu.edu
}

\author{
Michael L. Nelson \\ Old Dominion University \\ Norfolk, VA, USA \\ mln@cs.odu.edu
}

\begin{abstract}
When users post links to web pages in Twitter there is a time delta between when the post was shared $\left(t_{\text {tweet }}\right)$ and when it was read $\left(t_{c l i c k}\right)$. Ideally, when this time delta is small there is often no change in the page's state. However upon reading shared content in the past and due to the dynamic nature of the web, the page's state could change and the intention of the author need to be inferred. In this work, we enhance a prior temporal intention model and tackle its shortcomings by incorporating extended linguistic feature analysis, replacing the prior textual similarity measure with semantic similarity one based on latent topic detection trained on Wikipedia English corpus, and finally by enriching and balancing the training dataset. We uncovered three different intention behaviors in respect to time: Stable Intention, Changing Intention from current to past, and Undefined intention. Using these classes and only the information available at posting time from the tweet and the current state of the resource, we correctly predict the temporal intention classification and strength with $77 \%$ accuracy.
\end{abstract}

\section{INTRODUCTION}

When a link to a webpage is shared in social media, authors have an implicit expectation as to what people in their social network would read when they click on that link. Authors might want the readers to view the exact same version of the page they saw when they posted (at time $t_{\text {tweet }}$ ); or they might want the readers to examine the most current version of the resource at the time of clicking on the link $\left(t_{\text {click }}\right)$. If the time difference is small $\left(t_{\text {click }}-t_{\text {tweet }} \approx 0\right)$, then the reader will not notice any discrepancy or shift in what the author intended for them to see. Unfortunately, due to the highly dynamic nature of the web it is highly likely that the intentions diverge through time either by change or loss of resources. This divergence could be highly problematic upon archiving and curating social media posts that narrate public events like the Arab Spring, Occupy Wall Street

Permission to make digital or hard copies of all or part of this work for personal or classroom use is granted without fee provided that copies are not made or distributed for profit or commercial advantage and that copies bear this notice and the full citation on the first page. Copyrights for components of this work owned by others than ACM must be honored. Abstracting with credit is permitted. To copy otherwise, or republish, to post on servers or to redistribute to lists, requires prior specific permission and/or a fee. Request permissions from permissions@ acm.org. JCDL'15, June 21-25, 2015, Knoxville, Tennessee, USA. Copyright (C) 2015 ACM 978-1-4503-3594-2/15/06 ...\$15.00. DOI: http://dx.doi.org/10.1145/2756406.2756921. movement, natural disasters like Hurricane Sandy and others.

GCL. Saunders
@sconsult
interest, the WHO has been releasing
regular flu A(H1N1) updates, latest is \#47
http://bit.ly/whodu
10:46 AM - 11 Jun 2009

(a) The intention is towards the past version at $t_{\text {tweet }}$.

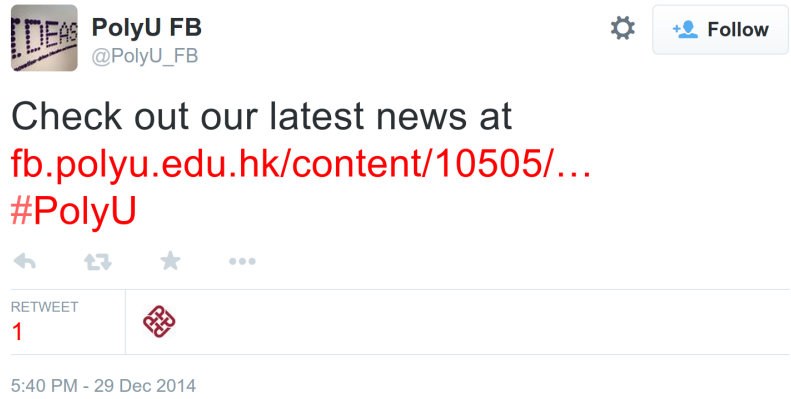

(b) The intention is towards the latest version at $t_{c l i c k}$.

Figure 1: Tweet examples for different intention classes.

Consider the tweets shown in Figure 1. In Figure 1(a) we can see that the author's intention is for a specific information resource and thus the intention is $t_{\text {tweet }}$. In Figure 1(b) the author wants the reader to see the latest information, so the intention is $t_{c l i c k}$. In this work, we build a predictive model which can effectively differentiate between each intention class at tweet-authoring time. The ability to differentiate the intention in real-time can be used to push a copy of the linked resource into a web archive (e.g., webcitation.org, archive.today, archive.org) at $t_{\text {tweet }}$ so the link is to an archived version instead of a web version, thus ensuring what readers see is consistent with the author's intention.

In our prior work, we analyzed the temporal intention concept and were able to successfully model temporal intention by breaking it down into two simpler tasks of content relevance and change, and named it the Temporal Intention Relevance Model (TIRM) [17]. Relevance describes whether 
the content of the resource still matches the tweet in topicality and context, while Change means that the resource have changed in content from the initial state at the time of tweeting. The subproblems of relevance and change are more straightforward to measure and there is an abundance of prior literature addressing them in regards to textual content relevance, as well as linguistic and semantic similarity.

To illustrate TIRM, we examine two points in the life of a tweet as described earlier: 1) $t_{\text {tweet }}$ when the author of the tweet posted it, 2) $t_{\text {click }}$ when the reader clicks on the link to examine the resource at current time. Table 1 shows that if the resource has changed and no longer relevant, then the intention is for the past (e.g., in Figure 1(a) the author intends for readers to access the WHO page as it was at $\left.t_{\text {tweet }}\right)$, while if the resource changed but still relevant, then the intention is for the current (e.g., in Figure 1(b) the author intends for readers to access latest news page as it will be at $\left.t_{c l i c k}\right)$. The model was trained using 39 different social, archival, and textual features extracted at $t_{c l i c k}$.

\begin{tabular}{rc|c} 
Resource's State & \multicolumn{2}{c}{ Tweet and resource are: } \\
Changed & Relevant & Not Relevant \\
\cline { 2 - 3 } Not Changed & $t_{\text {click }}$ & $t_{\text {tweet }}$ \\
\cline { 2 - 3 } Cheet & either or undefined \\
\cline { 2 - 2 } &
\end{tabular}

Table 1: Temporal Intention Relevancy Model (TIRM).

When we first started using TIRM in prediction we noticed that our model in its simplistic form had some shortcomings. One problem in judging relevance between a tweet and a linked resource is the tweet is limited to 140 characters while the resource could span thousands of characters. This highlights the need to find a better similarity measure based on the semantic similarity rather than just textual overlap. Second problem is that the model is more aggressive towards relevance due to the bias in the training dataset extracted from Mechanical Turk (80\% Relevant class and 20\% NonRelevant). Therefore, prior to the prediction analysis steps we extend the model to address those problems and enhance the model.

Following the enhancement of the model, we wanted to estimate the confidence of this probabilistic classification. We are able to tell the relevance class and if the content is changed or not, but not a collective classification measure of confidence. We propose a formulation of intention based on the relevance measure from the classifier and the change measure obtained by calculating similarity between the resource's versions. We call this formulation the Intention Strength Measure.

With this measure, for each resource in our dataset we calculate the intention measure at 12 points in the time period spanning 3.5 years from $t_{\text {click }}$ to $t_{\text {tweet }}$. Plotting those intention strength across time We devised a model to identify the intention progression from $t_{c l i c k}$ to $t_{\text {tweet }}$. This modeling will enable us to predict this temporal intention at an early stage, and provide the authors with enough information to perform an educated assessment on how to successfully convey their intention to the readers at any future $t_{c l i c k}$. For example, in the tweet shown in Figure 1, at $t_{\text {tweet }}$ when the author is posting a tweet with a link to the WHO Disease Outbreak News page, if it was predicted that the intention would change in the future they might push a copy of the current state of the page into one of the public archives and link to that snapshot instead of the live version.

\section{RELATED WORK}

Researchers also explored the problem of web content changing through time and how to predict this change $[1,4,5,8]$. Radinsky et al. introduced a new method incorporating not only the analysis of the page changes in the past but also the usefulness of this change and how related this page under observation is to other pages and how similar they are in the types of changes they undergo [14].

Latent Dirichlet Allocation (LDA) has been used extensively in detecting latent relations between phrases and words in corpora. Rus et al. investigated LDA and Latent Semantic Analysis (LSA) in deriving meaning on word level and phrase level in large corpora [16, 12]. In conversational analysis, and perhaps in Twitter, Oliva et al. explored the concept of semantic similarity in relatively short-text and built their method based on the semantic meaning behind words along with the structural relation between those words in the short text [13]. The semantic understanding of text and the meaning behind it could be utilized in further more than textual similarity but rather in identifying usefulness of content posted in social media [11]. Beyond merely textual analysis, Chen et al. have focused on analyzing the tweets that are associated with other resources, namely image tweets [3]. In their search for content relevancy between the associated images and the text, they explored various textual, visual, and social context features. They utilized LDA in modeling and detecting textual and visual hidden topics.

LDA has been also utilized in other context as well like tweet classification. The concept of retweeting was analyzed by Wang et al. in their search for the linguistic and functional factors leading to retweeting [20]. In their work they analyzed a multitude of factors in the tweet's structure and text ranging from sentiment analysis, emoticons existence, part-of-speech (POS) tagging, named entity extraction (NE), hashtag analysis, verb tense analysis, discourse relations and phrase similarity. In our work, we adopt their approach in tweet analysis and extract similar features aiding the enhancement of our model.

\section{ENHANCING TIRM}

To enhance TIRM we focused on the shortcomings of the model in regards to linguistic analysis, similarity measures, and dataset balancing while utilizing the same dataset used in TIRM spanning 1,124 instances.

\subsection{Linguistic Feature Analysis}

Previously, 39 different features were extracted from the tweet-resource pair in regards to similarity, URL structure, social and archival existence. The results were promising but we needed a deeper analysis and understanding of the linguistic properties of the tweet-resource pair.

\subsubsection{Tweet structural analysis}

After filtering out the linked resource we checked the existence of user mentions, hashtags, question marks "?" (indicating a question tweet), and exclamation marks "!" (indicating an expression of strong feelings). Furthermore we utilized regular expressions, adopted from Ritter et al.'s work, 
in detecting emoticons in the tweets [15]. We deduced that along with the extracted sentiment from the prior experiment we will be able to capture the emotional state the author. Finally, we also checked if the tweet was a re-tweet. These simple features proved to be highly effective as six of which are present among the top 13 ranked features in information gain of the retrained model as shown by table 3 (Key: $\mathrm{FB}=$ Facebook, Twt $=$ Tweet, $\mathrm{Sim}=$ Similarity, Cur=Current, Len $=$ Length, Celeb $=$ Celebrities, Pct $=$ Percent, Init $=$ Initial, $\mathrm{Pos}=$ Positive, $\mathrm{Neg}=$ Negative, $\mathrm{Neu}=$ Neutral).

\subsubsection{POS tagging and Named Entity Extraction}

In the prior TIRM, Wikipedia was harvested for lists of artists, actors, and singers from the English speaking world to use in detecting the existence of celebrities in the tweets. This feature proved to be highly valuable due to its corresponding high information gain. This observation led us to believe we need to further investigate named entities in tweets.

In tweet analysis, due to the rather small size of text with the lack of context and the informality in writing, tasks like Part-of-speech (POS) tagging, sentence chunking, and named entity recognition are quite challenging. Ritter et al. developed a distantly supervised approach that is tailored for tweet based analysis overcoming those challenges [15]. We adopted their labeled LDA-based POS tagger and chunker which have performed effectively against standard POS taggers on tweet datasets. Ten different types of entities are defined by Ritter's tagger as shown in table 2 along with the number of identified entities in each class across the training dataset of 1,124 instances. Furthermore, with the extracted POS tags and chunks we were able to determine if the most prominent tense in a tweet is present or past and used it as a feature too. The rationale behind this analysis is to also identify the intention of the author in discussing contemporaneous events or past ones.

\subsubsection{Tweet Classification}

Users tweet to convey an opinion, update a status, ask for information, sarcasm, spread jokes, and many other reasons [7]. In our search for the author's temporal intention we utilized Wang et al. work in classifying tweeting motive [20]. We adopted the first level of their two-tiered classification: Opinion, Update, Interaction, Fact, Deals, News, Others. Furthermore, and for the sake of simplicity, we utilized the largest classes of Opinion, Update, Interaction, Others which collectively comprised $94 \%$ of the instances in Wang et al.'s datset.

\begin{tabular}{c|c|c|c|}
\multicolumn{1}{c}{ Relevant } & \multicolumn{1}{c}{ Interaction } & \multicolumn{1}{c}{ Update } & \multicolumn{1}{c}{ Opinion } \\
\cline { 2 - 4 } Ron-Relevant & $69.67 \%$ & $59.28 \%$ & $36.99 \%$ \\
\cline { 2 - 4 } Non- & $30.33 \%$ & $40.72 \%$ & $63.01 \%$ \\
\cline { 2 - 4 } & &
\end{tabular}

Table 4: Tweet classification across relevancy classes
As shown in table 4, for class Interaction, the Relevance class is significantly higher than the other while in class Opinion the instances are more biased towards the NonRelevant. This indicates the relation between tweet class and relevance thus we use it as a feature.

\subsection{Semantic Similarity Analysis Using Latent Topic Modeling}

In the prior TIRM, similarity measures were based on word overlap either by using SimHash or n-grams cosine similarity. This technique proved to be lacking upon calculating the similarity between a tweet (140 characters) and a resource which could be virtually unlimited. Similarly between two versions of a resource, where a change in the html design could be interpreted as a low similarity while in fact the content itself remained unchanged. It is worth mentioning that in this experiment we only account for non-missing resources (i.e., gives 200 OK response). In their prior experiment, we attempted to overcome the latter problem by introducing a boiler plate removal algorithm to remove the effect of change in styling and extract the main content.

To address the former we employed topic detection, as we would consider a tweet and a resource to be similar if they were both mentioning the same topic, or discussing the same point. Thus we measure similarity based on collective semantics or "aboutness" of the pair rather than textual overlap.

We use both LSA and LDA in calculating the similarities between the tweet-resource and resource-resource pairs accordingly. We considered both techniques as LSA is much faster to train while LDA has higher accuracy. We also considered utilizing Twitter based LDA models from the works of Mehrotra et al. and Zhao et al. which are more fitted to handle tweeted textual content with its embedded hashtags $[10,21]$. Since we were not performing topic modeling on tweets only and we are calculating similarities between the tweet and the resource, which is written formally in most cases, traditional LDA-LSI models trained on a diverse corpus like Wikipedia seemed more suitable. Furthermore, we calculate the similarities between the resource versions (at $t_{\text {tweet }}$ or past version, and at $t_{\text {click }}$ or current version) and the tweet.

To prepare these models we utilize the Wikipedia Corpus in extracting the topics and features. We downloaded 4,295,020 documents spanning the English Wikipedia documents in January $2014^{1}$. We chose Wikipedia for training as it spans a wide variety of topics and the latent relations between them. Next we built the LDA and LSA models with 100,000 features, $672,235,199$ non-zero entries in the sparse TF-IDF matrix. The LDA model in this case is an online learning LDA model developed by Hoffman et al. [6]. We get the current version of the resource, the past version, and the tweet. We convert each to latent vector space and using the model we calculate the cosine similarity. The result is

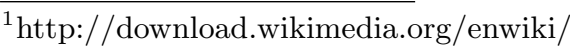

\begin{tabular}{|l|l|l|l|l|l|l|l|l|l|}
\hline Person & GeoLoc & TVShow & Movie & Facility & Company & Product & SportsTeam & Band & Other \\
\hline 233 & 81 & 18 & 37 & 19 & 115 & 42 & 10 & 62 & 96 \\
\hline \hline \multicolumn{3}{|c|}{ Tweets with Named Entities } & 543 & \multicolumn{3}{c|}{ Tweets without Named Entities } & 581 \\
\hline
\end{tabular}

Table 2: Named Entities instances in the dataset 


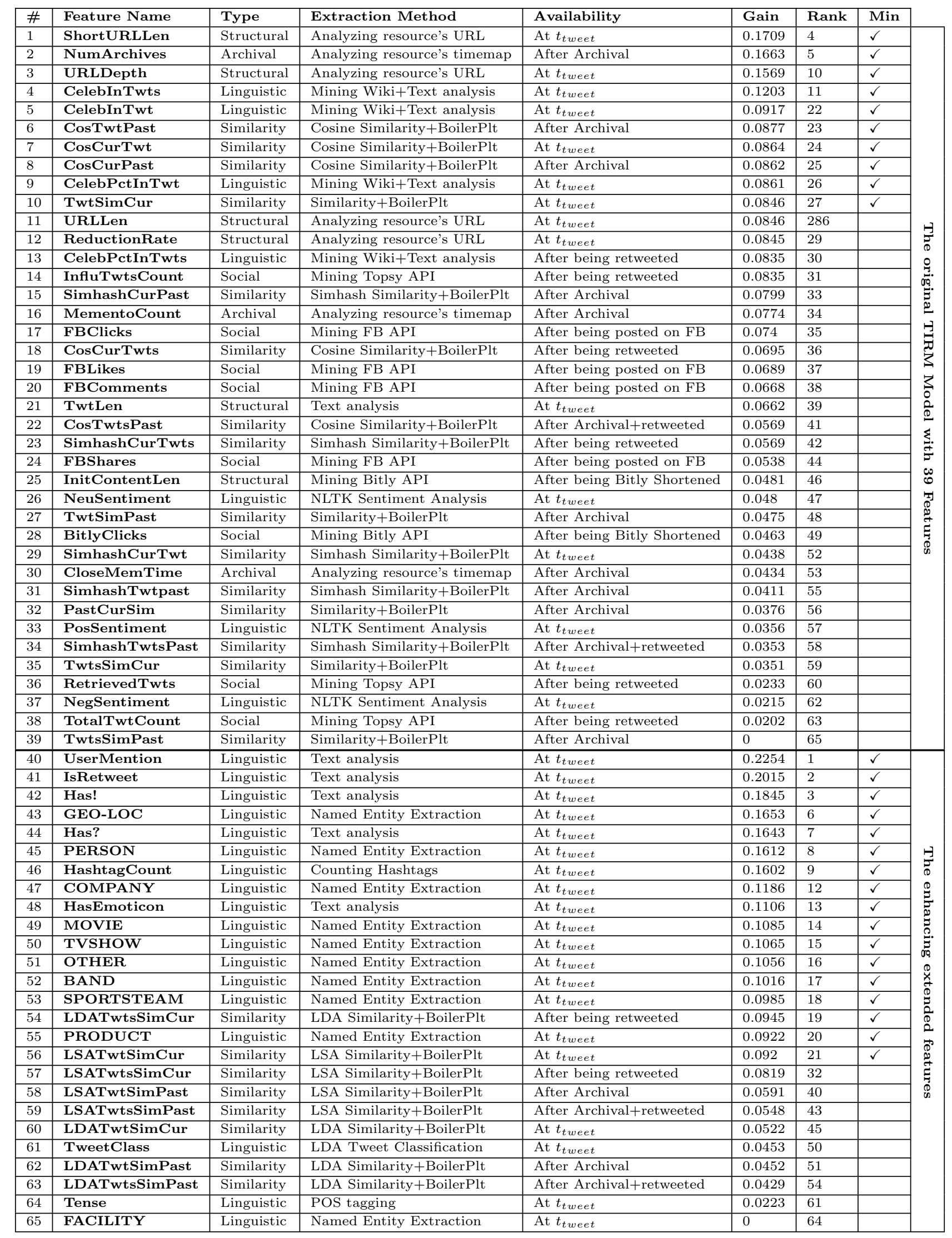

Table 3: All the features used in TIRM and after enhancement, ranked by Information Gain Ratio. 
a number ranging from 0 (indicating no similarity) and 1 (identical similarity). Gensim by Řehưřek et al. was used in our LDA and LSA modeling and similarity calculations [19].

\subsection{Dataset Balancing}

From the prior experiment, the dataset used in training and cross validation was collected using five different Mechanical Turk voters for 1,124 instances. The instances were classified by the majority of voters to Relevant and NonRelevant classes. Unfortunately, but yet matching intuition, the dataset collected is biased towards Relevancy (with 930 Relevant Vs. 194 Non-Relevant). This undersampling of the class Non-Relevant is causing the trained model to be more aggressive towards the Relevant class as shown in the class-based recall, precision and F-measure in table 6 .

The problem of imbalanced training datasets in classification is a well-known problem. In a multitude of cases, one of the classes is significantly lower in training points than the other class(es). This causes the classifier to be overly sensitive towards one class than the other. In our analysis, the Relevant class is almost five times in magnitude than the Non-Relevant class resulting in a reduced precision and recall in the minor class. Possible solutions to this problem is by undersampling the major class (Relevant) to be nearly the same size of the minor class (Non-Relevant). This approach has a downside as we purposely dispose of good data points that could enhance the classifier. Also it reduces the size of the training dataset for the collective classes gravely.

Another approach is the Synthetic Minority Over-sampling Technique or (SMOTE) introduced by Chawla et al. [2]. By synthesizing balancing datapoints via over-sampling the minor class in the dataset and utilizing the k-nearest neighbors algorithm, they were able to enrich the training dataset iteratively by oversampling the minor class until the two classes were close in size. Their technique proved to achieve better classifier performance (in ROC space) than undersampling the major class. Given so, we utilized SMOTE with five nearest neighbors in balancing our Relevant-NonRelevant dataset iteratively then we randomized the dataset uniformly. Table 5 shows the results of retraining the classifier after enhancement.

\subsection{Feature Minimization}

To this point, we have collected 65 different features (39 original +26 new) to train TIRM. Due to the associated high cost of calculating all the features, we investigate the effect of feature minimization on the trained classifier.

For each feature, there are two important factors: cost (computational power and time) and effectiveness (information gain ratio). We will assume a uniform cost and optimize in regards to information gain. We use ranker algorithm in extracting the top 25 features (as shown in table 3) in terms of information gain to retrain TIRM. Table 6 shows the $60 \%$ reduced TIRM classifier has a performance reduction of about $2 \%$.

\section{INTENTION STRENGTH}

To indicate the intention class, we use the resulting relevance from the model along with change in TIRM (as illustrated in table 1). This mapping model is effective, but

\begin{tabular}{|l|l|l|l|}
\cline { 2 - 4 } \multicolumn{1}{c|}{} & Precision & Recall & F-measure \\
\cline { 2 - 4 } \multicolumn{1}{c|}{ Prior TIRM } \\
\hline Relevant & 0.863 & 0.971 & 0.914 \\
\hline Non-Relevant & 0.654 & 0.263 & 0.375 \\
\hline Weighted Avg. & 0.827 & 0.849 & 0.821 \\
\hline
\end{tabular}

TIRM after Enhancement

\begin{tabular}{|l|l|l|l|}
\hline Relevant & 0.880 & 0.932 & 0.905 \\
\hline Non-Relevant & 0.928 & 0.873 & 0.900 \\
\hline Weighted Avg. & 0.904 & 0.903 & 0.903 \\
\hline
\end{tabular}

TIRM after Minimization

\begin{tabular}{|l|l|l|l|}
\hline Relevant & 0.849 & 0.939 & 0.892 \\
\hline Non-Relevant & 0.932 & 0.834 & 0.880 \\
\hline Weighted Avg. & 0.890 & 0.886 & 0.886 \\
\hline
\end{tabular}

Table 6: Results from the previous TIRM, TIRM after enhancement, and minimization with Random Forrest Classifier

unfortunately, although we can deduce the intention class (being past or current) but there is no quantification of this intention strength. To overcome this, we devise a formulation of calculating the intention strength in terms of change and relevance as follows.

For each resource $r$, the similarity $\sigma_{\text {past-current }}$ is calculated using LDA similarity illustrated earlier between the two versions, the past version (at $t_{\text {tweet }}$ ) and the current version (at $\left.t_{\text {click }}\right)$. Thus, $\delta_{\text {past-current }}$ is the normalized change ranging from 0.0 to 1.0 . From the classifier we extract the relevance measure $\rho(r)$ ranging from 0.0 - 1.0, where 0.0 being completely Non-Relevant, and 1.0 being completely Relevant. Referring back to the TIRM model table 1 we define the intention class $\chi(r)$ in terms of change $\delta(r)$ and relevance $\rho(r)$ as follows:

$$
\chi(r)= \begin{cases}\text { Current, } & \text { if } \rho(r)>0.5 \quad \& \quad \delta(r)>0.5 \\
\text { Past, } & \text { if }\left\{\begin{array}{lll}
\rho(r)<0.5 & \& & \delta(r)>0.5 \\
\rho(r)>0.5 & \& & \delta(r)<0.5
\end{array}\right. \\
\text { Unknown, } & \text { otherwise }\end{cases}
$$

After identifying the intention class $\chi(r)$, we calculate the intention magnitude or strength $|\chi(r)|$. From Figure 2 we can deduce that the point $\left(\rho\left(r_{s}\right), \delta\left(r_{s}\right)\right)=(1,1)$ means it is most relevant and completely changed which indicates the strongest "decided current intention" or $\left|\chi\left(r_{s}\right)\right|=1$.

Point $\left(\rho\left(r_{c}\right), \delta\left(r_{c}\right)\right)=(0.5,0.5)$ is considered the point of confusion as it illustrates peak uncertainty of intention, or $\left|\chi\left(r_{c}\right)\right|=0$. The further the new resource $(\rho(r), \delta(r))=$ $(\mathrm{x}, \mathrm{y})$ from the point of confusion the stronger the intention certainty is. The furthest distance is the distance from the confusion point $\left(\rho\left(r_{c}\right), \delta\left(r_{c}\right)\right)=(0.5,0.5)$ to certainty point $\left(\rho\left(r_{s}\right), \delta\left(r_{s}\right)\right)=(1,1)$. This distance $S$ will be used for normalization. Also the distances are Euclidean.

So to calculate $|\chi(r)|$ for the new resource $(\rho(r), \delta(r))=$ $(\mathrm{x}, \mathrm{y})$ we follow equation 2 .

$$
|\chi(r)|=\frac{L}{L^{\prime}}=\frac{\sqrt{\left(\rho(r)-\rho\left(r_{c}\right)\right)^{2}+\left(\delta(r)-\delta\left(r_{c}\right)\right)^{2}}}{\sqrt{\left(\rho\left(r_{s}\right)-\rho\left(r_{c}\right)\right)^{2}+\left(\delta\left(r_{s}\right)-\delta\left(r_{c}\right)\right)^{2}}}
$$


10-Fold Cross-Validation Testing

\begin{tabular}{|c|c|c|c|c|c|}
\cline { 2 - 6 } & \multicolumn{1}{c|}{$\begin{array}{c}\text { Mean } \\
\text { Absolute Error }\end{array}$} & $\begin{array}{c}\text { Relative } \\
\text { Absolute Error }\end{array}$ & $\begin{array}{c}\text { Kappa } \\
\text { Statistic }\end{array}$ & $\begin{array}{c}\text { Incorrectly } \\
\text { Classified \% }\end{array}$ & $\begin{array}{c}\text { Correctly } \\
\text { Classified \% }\end{array}$ \\
\cline { 2 - 6 } Enhanced TIRM & 0.22 & $75.77 \%$ & 0.31 & $15.12 \%$ & $84.88 \%$ \\
\cline { 2 - 6 } & 0.20 & 39.69 & 0.81 & $9.73 \%$ & $90.27 \%$ \\
\hline
\end{tabular}

Table 5: Results of 10-fold cross-validation for TIRM prior and after the three-staged enhancement process.

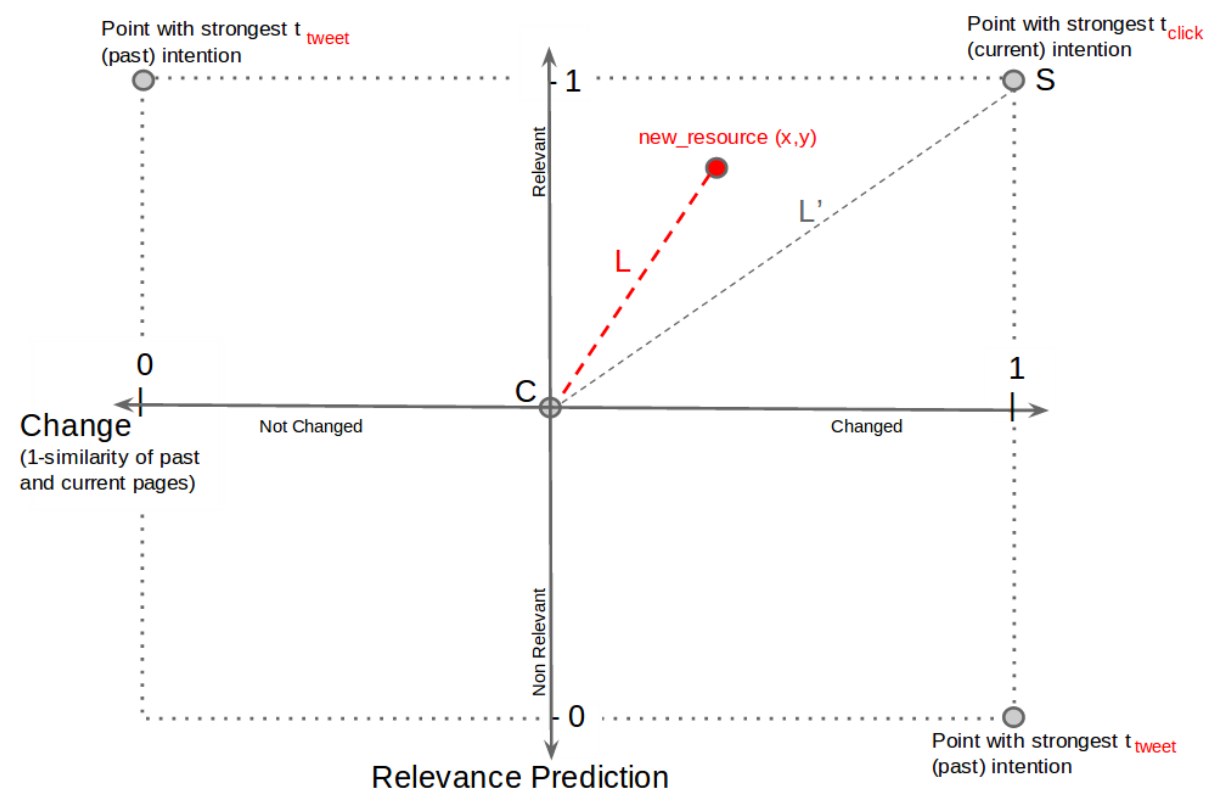

Figure 2: Intention Strength Mapping.

Or to simplify: strengthsInstances

$$
\begin{gathered}
|\chi(r)|=\frac{\sqrt{\left(\rho(r)-\frac{1}{2}\right)^{2}+\left(\delta(r)-\frac{1}{2}\right)^{2}}}{\sqrt{\left(1-\frac{1}{2}\right)^{2}+\left(1-\frac{1}{2}\right)^{2}}} \\
|\chi(r)|=\sqrt{2\left[\left(\rho(r)-\frac{1}{2}\right)^{2}+\left(\delta(r)-\frac{1}{2}\right)^{2}\right]}
\end{gathered}
$$

Finally by merging the intention class $\chi(r)$ and the intention strength $|\chi(r)|$ we get this formula:

$$
|\chi(r)|=\left\{\begin{aligned}
|\chi(r)| & \text { if } \chi(r) & =\text { Current } \\
-|\chi(r)| & \text { if } \chi(r) & =\text { Past } \\
\text { Undefined } & \text { if } \chi(r) & =\text { Unknown }
\end{aligned}\right.
$$

Equation 5 summarizes $|\chi(r)|$ to be a value ranging from -1.0 to 1.0 , with -1.0 being the strongest Past intention and 1.0 being the strongest Current intention. For the 1,124 instances in the dataset we calculate the corresponding intention strengths $\left|\chi\left(r_{1-1,124}\right)\right|$. Figure 3 shows a histogram of the instances in each intention strength bin ranging from -1.0 to 1.0 and Figure 4 shows the sorted instances in terms of intention strength.

\section{MODELING INTENTION ACROSS TIME}

At this point, we are able to calculate the intention strength at the current time, and whether it is current or past, given

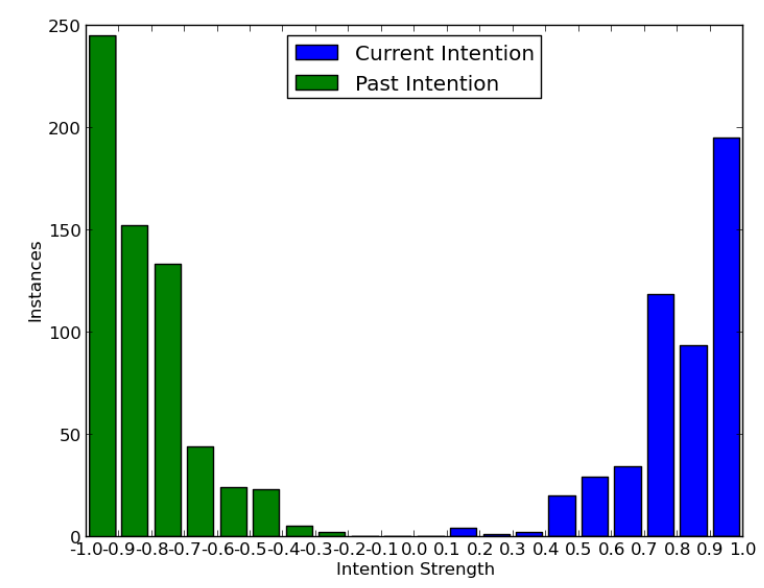

Figure 3: Histogram of the 1,124 instances in each intention strength bin.

the tweet and the resource's state at $t_{\text {tweet }}$ and at $t_{\text {click }}$. The next logical question was: Did the intention strength through the life span of the resource between $t_{\text {tweet }}$ and $t_{\text {click }}$ change at one point during these three and half years?

Answering this question will put us on track of answering the ultimate question of this paper: Would the study of how intention strength change through time give us a way 


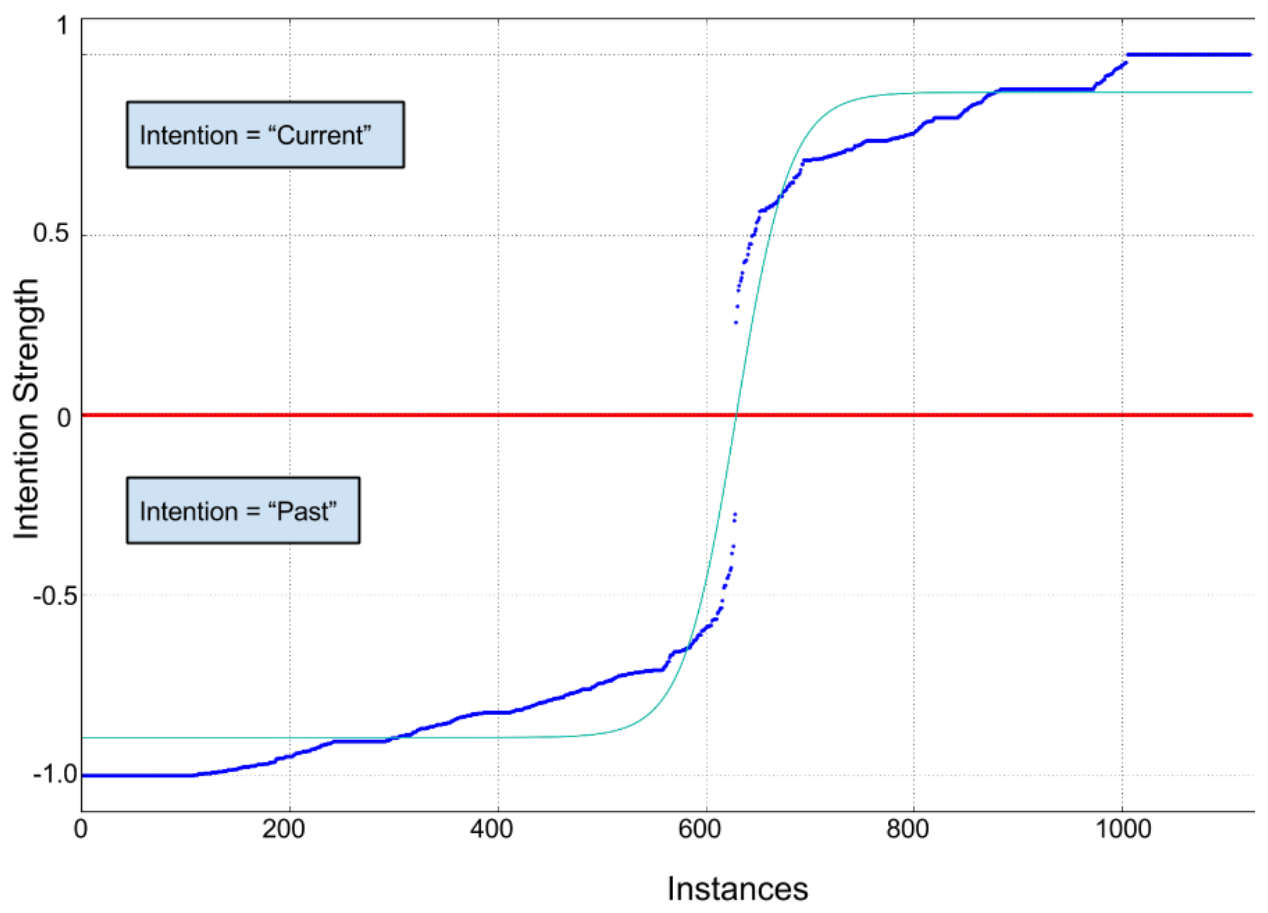

Figure 4: Intention strength across all 1,124 instances

\begin{tabular}{|c|l|}
\hline Behavior & \multicolumn{1}{c|}{ Example Tweet } \\
\hline Steady-Current & Check out our latest news at http://bit.ly/1xC7MhK \#PolyU \\
\hline Changing & $\begin{array}{l}\text { @heathermeeker The media just lost interest, the WHO has been releasing regular flu A(H1N1) } \\
\text { updates, latest is \#47 http://bit.ly/whodu }\end{array}$ \\
\hline Steady-Past & The Real Secret to Becoming a Popular Blogger http://bit.ly/16OY7q via @FreelanceSw \\
\hline
\end{tabular}

Table 7: Tweet examples of the behavior classes.

to predict this change at an earlier point in time, namely at posting time $t_{\text {tweet }}$ ?

In the dataset used in building the model, the 1,124 tweets were extracted from the Stanford Network Analysis Project (SNAP) Twitter dataset [9] which spanned around 476 million tweets. These instances were collected in the period from June to December of 2009. The Mechanical Turk experiment was conducted and the current snapshots were captured in January 2013 after about three and half years. We utilized the public archives through the Memento Framework to extract the state of the linked resource at the time of the tweet [18]. To get the past version of the resource, we extracted the closest memento to the time of posting the tweet $t_{\text {tweet }}$. To gauge the validity of the extracted past version, we measured the time difference between $t_{\text {tweet }}$ and the creation time of that closest memento. The time difference between $t_{\text {tweet }}$ and $t_{\text {closest_memento }}$ was ranging from 3.07 minutes to 56.04 hours averaging 25.79 hours. For the sake of simplicity, we assumed these time deltas are negligible and $t_{\text {closest_memento }} \approx t_{\text {tweet }}$. Following the same paradigm we extracted 10 mementos from the period between $t_{\text {tweet }}$ and $t_{\text {click: }}$ :

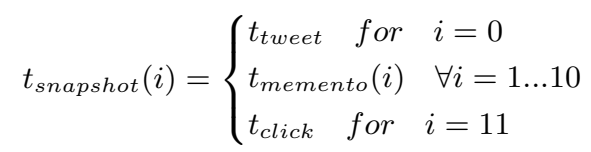

Where $i=0$ means the first snapshot which is at time of the tweet and $i=11$ means the last snapshot at the current end time of the experiment. The ten downloaded mementos are at $i=1 \rightarrow 10$.

The next step is to calculate the intention strength at each of those 12 points in time. Since we need to simulate the state at each time $t_{\text {snapshot }}(i)$ we need to download the state of the resource, get the Bitly clicklogs and the summation of the posted tweets up to this time. We mined the Bit.ly API to extract the clicks count to that moment $t_{\text {snapshot }}(i)$. We extracted the tweets posted till $t_{\text {snapshot }}(i)$ from Topsy.com API. This is another rationale behind using Topsy API instead of the Twitter API as the latter does not enable searching further than the indexing period (two weeks). Furthermore, we calculated all the applicable features for each snapshot as shown in Figure 5. Finally, using our prior trained model and the strength formulation we calculated $\left|\chi\left(r_{i}\right)\right|$ for each snapshot and plotted them across time as shown in examples in Figure 6.

\section{PREDICTING TEMPORAL INTENTION AT TWEET TIME}

In Figure 6, the blue points indicate the intention strength at this point in time. We noticed a steady behavior with respect to time in some cases and a changing behavior in others. This matches our intuition that users intended for the 


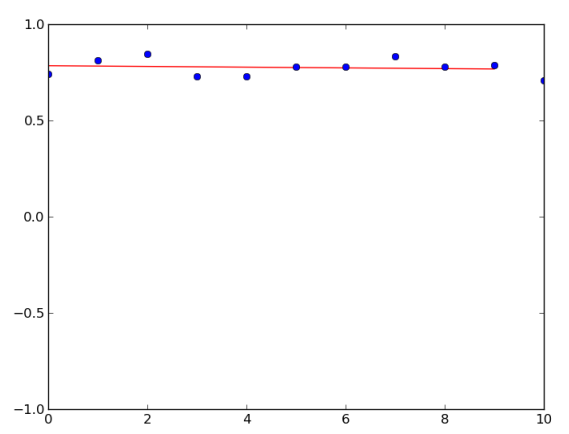

(a) Steady Current intention: $\sim 0$ slope.

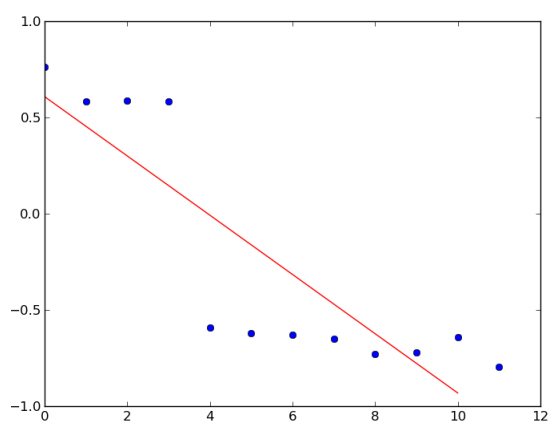

(b) Changing intention: Current to Past, -ve slope.

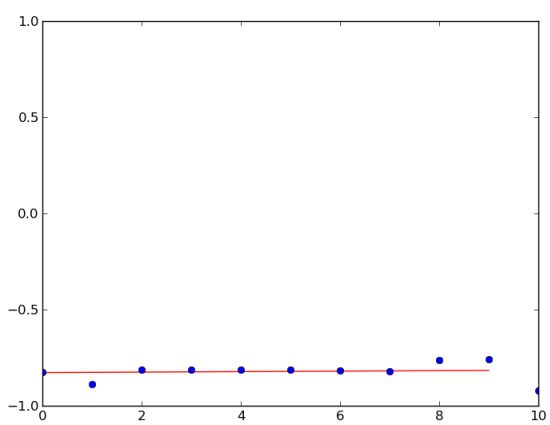

(c) Steady Past intention: 0 slope.

Figure 6: The resources' intention strength across time for different behavior categories.

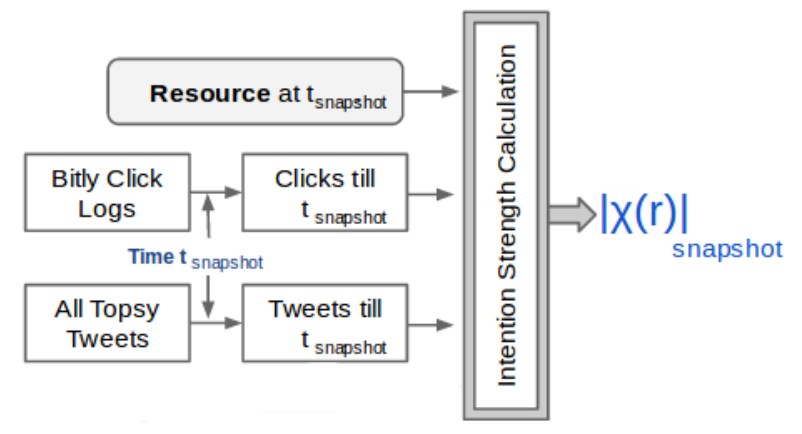

Figure 5: Intention Strength calculation per snapshot

readers to see the version at $t_{\text {click }}$ for the first short period of time, but upon changing and updating of the resource the intention deviated to the $t_{\text {tweet }}$ version as shown in Figure 6(b).

To further analyze this phenomena, and to differentiate the steady state from the changing one, we fitted with blue intention strength points in the graphs with the closest linear regression line (red line) to measure its progression through its slope as shown as well in Figure 6. Evidently, if the slope was negative this indicate the intention has changed from current to past. We use both the slope of the fitted regression line and the fitting error to cluster the plots into three different categories: Steady, Changing, and Unknown. The Steady Intentional behavior means the slope is small and the fitting error is small, this indicates a resource where the intention did not change across time. The Changing Intentional behavior means the slope is negative indicating a change in intention from current to past across time, with a moderate fitting error. Finally the Unknown Intentional behavior is where the regression line fitting error is too high or the 4th class of our TIRM model where the resource is not relevant and did not change.

Given the slope, intercept, and fitting error along with the other features, we were able to successfully train a regression classifier to automatically categorize the behavior of a resource across time into either one of these three categories. We performed a 10-fold cross-validation and the classifier correctly classified $89 \%$ of the dataset as shown in table 9 . We were able to identify the behavioral class of in- tention given the knowledge of the state of the resource and the social network around it through time; The next step is to validate the viability of identifying these classes given only the information available at tweet time $t_{\text {tweet }}$.

With our model from the previous stage, we filtered out all the longitudinal temporal features and kept only the features extracted from the tweet and the current version of the resource at $t_{\text {tweet }}$. We retrained the classifier using these limited features and it correctly classified $77 \%$ of the dataset. This percentage is lower than the prior percentage with the full knowledge of the resource in time as expected. Moreover, it is still high enough indicating the viability of predicting the temporal intention progression given only the knowledge of the tweet at posting time and the state of the resource at $t_{\text {tweet }}$ as shown also in tables 8 and 9 .

In other words, at the time of authoring a tweet, and given only the information about the resource and the tweet available at the current time, we can predict for the author whether the intention conveyed to the readers will be consistent or will it change with $77 \%$ accuracy.

Returning to our tweet examples, in the tweet in Figure 1(a), the model predicted a change in intention from current to past with $60 \%$ probability. While in the tweet in Figure 1(b), the model predicted a $60 \%$ probability of steady-current intention. Furthermore in table 7 , for the third tweet our model predicted a steady behavior with a $40 \%$ probability.

This prediction will give the author sufficient information to choose to just post the tweet or take a snapshot of the resource and push it into one of the public archives and link to that snapshot instead to maintain the consistency. This prediction will have implication on maintaining the consistency of the conveyed information on the web and will help enrich the archived content of a multitude of resources by crowdsourcing the preservation task.

\begin{tabular}{|l|l|l|l|}
\cline { 2 - 4 } \multicolumn{1}{c|}{} & Precision & Recall & F-measure \\
\hline Steady Intention & 0.680 & 0.715 & 0.697 \\
\hline $\begin{array}{l}\text { Changing Intention } \\
\text { (Current to Past) }\end{array}$ & 0.912 & 0.897 & 0.904 \\
\hline Undefined Intention & 0.713 & 0.688 & 0.700 \\
\hline Weighted Avg. & 0.768 & 0.767 & 0.767 \\
\hline
\end{tabular}

Table 8: Intention behavior prediction classifier 
10-Fold Cross-Validation Testing

\begin{tabular}{|c|c|c|c|c|c|}
\cline { 2 - 5 } Predicting Intention & $\begin{array}{c}\text { Mean } \\
\text { Absolute Error }\end{array}$ & $\begin{array}{c}\text { Relative } \\
\text { Absolute Error }\end{array}$ & $\begin{array}{c}\text { Kappa } \\
\text { Statistic }\end{array}$ & $\begin{array}{c}\text { Incorrectly } \\
\text { Classified \% }\end{array}$ & $\begin{array}{c}\text { Correctly } \\
\text { Classified \% }\end{array}$ \\
\cline { 2 - 6 } With all Features & 0.15 & $34.11 \%$ & 0.84 & $10.94 \%$ & $89.06 \%$ \\
\cline { 2 - 6 } $\begin{array}{c}\text { With the tweet and } \\
\text { the resource at } t_{\text {tweet }}\end{array}$ & 0.22 & $50.57 \%$ & 0.65 & $23.32 \%$ & $76.68 \%$ \\
\cline { 2 - 6 } & & &
\end{tabular}

Table 9: Results of 10-fold cross-validation for predicting intention behavior strength across time.

Tweet's Intention Prediction
Place in the text box the tweet you which to predict its author's temporal intention. The
tweet must have a "http://bitly" shortened URL.
Tweet:
Predict

Figure 7: Intention Oracle API service

\section{INTENTION ORACLE API}

We built a proof-of-concept class prediction service which implements the prediction model described in section 6 . The service takes a tweet with a URL shortened via Bit.ly and extracts the necessary features after downloading content and then predicts the behavioral class of the tweet. For the time being it classifies if the resource is more likely to be in a steady state of intention or a changing state of intention. The service interface is shown in Figure 7 and a sample JSON-encoded response obtained in correspondence to the three tweet examples in table 7 respectively are demonstrated in Figure 8.

\section{CONCLUSIONS}

In this work, we analyze the problem of temporal intention in sharing resources in social media. We adopt a prior preliminary model in analyzing users' temporal intention. This model enabled us to detect and classify relevance and map it along with the resource's change to extract the intention class of the tweet in relation to the linked resource in it. In this paper, our first contribution is by enhancing this model and addressing the short comings in regards to linguistic features analysis, balancing the training dataset, and finally using latent semantics in measuring similarity instead of merely textual resemblance. With these three stages, we were able to enhance the model considerably, especially in the Non-Relevant class, with a 0.5 improvement in F-measure and a $6 \%$ increase in total classification from the prior model upon utilizing a Random Forrest based classifier.

Our second contribution is quantifying this temporal intention based on the enhanced model. We formulate a combination of the new semantic change measure and the relevance prediction from the enhanced classifier to produce a normalized quantifiable intention strength measure ranging from -1.0 to 1.0 (past to current intention, respectively).
The third contribution is analyzing the progression of intention through time. We simulate the intention analysis over the period of 3.5 years from June 2009 to January 2013 to observe the intention strength change across time. We observe three different classes of behavior: a steady intention either in the past or current, a changing intention gradually with time from the current to past, and undefined intention. We use these observations to fit regression lines to calculate the slopes and intercepts of intention to detect the progression scheme through time.

Our fourth contribution is in predicting the temporal intention at the time of authoring a tweet. We incorporate

\{

"Tweet Analyzed": "Check out our latest news at http://bit.1y/1xC7MhK \#PolyU", "Bitly Extracted": "http://bit.ly/1xC7MhK", "Original Resource URL": "http://www.fb. polyu.edu.hk/content/10505/index.html", "State": "Steady, Not changing", "Prediction": "Predicted Steady intention for the resource with $60.0 \%$ confidence", \}

\{

"Tweet Analyzed": "@heathermeeker The media just lost interest, the WHO has been releasing regular lu A(H1N1) updates, latest is \#47 http://bit.ly/whodu",

"Bitly Extracted": "http://bit.ly/whodu", "Original Resource URL": "http://www.who. int/csr/don/en/",

"State": "Unsteady, Changing",

"Prediction": "Predicted Unsteady intention observed for the resource, recommend preservation with $60.0 \%$ confidence", "Confidence": "60.0"

\{

"Tweet Analyzed": "The Real Secret to Becoming a Popular Blogger http://bit.ly/ 160Y7q via @FreelanceSw",

"Bitly Extracted": "http://bit.ly/160Y7q",

"Original Resource URL": "http://www. copyblogger.com/popular-blogger/", "State": "Steady, Not changing",

"Prediction": "Predicted Steady intention for the resource with $50.0 \%$ confidence", \}

"Confidence": "50.0"

Figure 8: JSON Objects resulting from the Intention Oracle API 
these features to our previous dataset across time and model the change of intention with a success of $89 \%$. Finally we predict this change or steadiness of intention at $t_{\text {tweet }}$ by using only the features that are readily available at $t_{\text {tweet }}$ from both the tweet and the resource and were able to successfully predict this intention with $77 \%$ accuracy. Giving the authors enough information to aid them to either re-write the tweet with the knowledge of change or push a snapshot of the resource to one of the public archives and link to it instead, maintaining temporal consistence and enriching the archives at the same time.

Finally we demonstrate a prototype API service implementation of our intention prediction model to be utilized on tweet with links shortened via Bitly.com service.

\section{ACKNOWLEDGMENT}

This work was supported in part by the Library of Congress and NSF IIS-1009392. We thank the anonymous reviewers for their suggestions in regards to the strength formulation.

\section{REFERENCES}

[1] E. Adar, J. Teevan, S. T. Dumais, and J. L. Elsas. The Web Changes Everything: Understanding The Dynamics Of Web Content. In WSDM '09: Proceedings Of The Second ACM International Conference On Web Search and Data Mining, pages 282-291, 2009.

[2] N. V. Chawla, K. W. Bowyer, L. O. Hall, and W. P. Kegelmeyer. SMOTE: Synthetic Minority Over-Sampling Technique. Journal Of Artificial Intelligence Research, 16(1):321-357, June 2002.

[3] T. Chen, D. Lu, M.-Y. Kan, and P. Cui. Understanding and Classifying Image Tweets. In Proceedings Of The 21st ACM International Conference On Multimedia, MM '13, pages 781-784, New York, NY, USA, 2013. ACM.

[4] J. Cho and H. Garcia-Molina. Estimating Frequency Of Change. ACM Transactions On Internet Technology, 3(3):256-290, Aug. 2003.

[5] D. Fetterly, M. Manasse, M. Najork, and J. Wiener. A Large-Scale Study Of The Evolution Of Web Pages. In WWW '03: Proceedings of The 12th International Conference On World Wide Web, pages 669-678, 2003.

[6] M. D. Hoffman, D. M. Blei, and F. R. Bach. Online Learning For Latent Dirichlet Allocation. In NIPS, pages 856-864, 2010.

[7] A. Java, X. Song, T. Finin, and B. Tseng. Why We Twitter: Understanding Microblogging Usage and Communities. In Proceedings Of The 9th WebKDD and 1st Sna-Kdd 2007 Workshop On Web Mining and Social Network Analysis, WebKDD/SNA-KDD '07, pages 56-65, New York, NY, USA, 2007. ACM.

[8] M. Klein and M. L. Nelson. Investigating The Change Of Web Pages' Titles Over Time. Technical Report arXiv:0907.3445, 2009.

[9] J. Leskovec and A. Krevl. Snap Datasets: Stanford Large Network Dataset Collection. http://snap.stanford.edu/data, June 2014.

[10] R. Mehrotra, S. Sanner, W. Buntine, and L. Xie. Improving Lda Topic Models For Microblogs Via
Tweet Pooling and Automatic Labeling. In Proceedings Of The 36th International ACM SIGIR Conference On Research and Development In Information Retrieval, SIGIR '13, pages 889-892, New York, NY, USA, 2013. ACM.

[11] E. Momeni, K. Tao, B. Haslhofer, and G.-J. Houben. Identification Of Useful User Comments In Social Media: A Case Study On Flickr Commons. In Proceedings Of The 13th ACM/IEEE-CS Joint Conference On Digital Libraries, JCDL '13, pages 1-10, New York, NY, USA, 2013. ACM.

[12] N. Niraula, R. Banjade, D. Stefanescu, and V. Rus. Experiments With Semantic Similarity Measures Based On Lda and Lsa. Statistical Language and Speech Processing, 7978:188-199, 2013.

[13] J. Oliva, J. I. Serrano, M. D. Del Castillo, and A. Iglesias. Symss: A Syntax-Based Measure For Short-Text Semantic Similarity. Data 85 Knowledge Engineering, 70(4):390-405, 2011.

[14] K. Radinsky and P. N. Bennett. Predicting content change on the web. In Proceedings of the Sixth ACM International Conference on Web Search and Data Mining, WSDM '13, pages 415-424, New York, NY, USA, 2013. ACM.

[15] A. Ritter, S. Clark, Mausam, and O. Etzioni. Named Entity Recognition In Tweets: An Experimental Study. In Proceedings Of The Conference On Empirical Methods In Natural Language Processing, EMNLP '11, pages 1524-1534, Stroudsburg, PA, USA, 2011. Association for Computational Linguistics.

[16] V. Rus, N. Niraula, and R. Banjade. Similarity Measures Based On Latent Dirichlet Allocation. Computational Linguistics and Intelligent Text Processing, 7816:459-470, 2013.

[17] H. M. SalahEldeen and M. L. Nelson. Reading The Correct History?: Modeling Temporal Intention In Resource Sharing. In Proceedings of The 13th ACM/IEEE-CS Joint Conference On Digital Libraries, JCDL '13, pages 257-266, New York, NY, USA, 2013. ACM.

[18] H. Van de Sompel, M. L. Nelson, R. Sanderson, L. Balakireva, S. Ainsworth, and H. Shankar. Memento: Time Travel For The Web. Technical Report arXiv:0911.1112, 2009.

[19] R. Řehuřek and P. Sojka. Software Framework For Topic Modelling With Large Corpora. In Proceedings Of The LREC 2010 Workshop On New Challenges For NLP Frameworks, pages 45-50, Valletta, Malta, May 2010. ELRA.

[20] A. Wang, T. Chen, and M.-Y. Kan. Re-Tweeting From A Linguistic Perspective. In Proceedings Of The Second Workshop On Language In Social Media, LSM '12, pages 46-55, Stroudsburg, PA, USA, 2012. Association for Computational Linguistics.

[21] W. X. Zhao, J. Jiang, J. Weng, J. He, E.-P. Lim, H. Yan, and X. Li. Comparing Twitter and Traditional Media Using Topic Models. In Proceedings Of The 33rd European Conference On Advances In Information Retrieval, ECIR'11, pages 338-349, Berlin, Heidelberg, 2011. Springer-Verlag. 